\title{
Glauber dynamics of continuous particle systems
}

\author{
Yuri Kondratiev $^{\mathrm{a}, \mathrm{b}, \mathrm{c}}$, Eugene Lytvynov ${ }^{\mathrm{c}, \mathrm{d}, \mathrm{e}, *}$ \\ ${ }^{a}$ Fakultät für Mathematik, Universität Bielefeld, Postfach 1001 31, 33501 Bielefeld, Germany \\ $\mathrm{b}$ Institute of Mathematics, Kiev, Ukraine \\ ${ }^{\mathrm{c}}$ BiBoS, Univ. Bielefeld, Germany \\ ${ }^{\mathrm{d}}$ Department of Mathematics, University of Wales Swansea, Singleton Park, Swansea SA2 8PP, Wales, UK \\ e SFB 611, Univ. Bonn, Germany
}

Received 20 June 2003; received in revised form 6 April 2004; accepted 26 May 2004

Available online 11 September 2004

\begin{abstract}
This paper is devoted to the construction and study of an equilibrium Glauber-type dynamics of infinite continuous particle systems. This dynamics is a special case of a spatial birth and death process. On the space $\Gamma$ of all locally finite subsets (configurations) in $\mathbb{R}^{d}$, we fix a Gibbs measure $\mu$ corresponding to a general pair potential $\phi$ and activity $z>0$. We consider a Dirichlet form $\mathcal{E}$ on $L^{2}(\Gamma, \mu)$ which corresponds to the generator $H$ of the Glauber dynamics. We prove the existence of a Markov process $\mathbf{M}$ on $\Gamma$ that is properly associated with $\mathcal{E}$. In the case of a positive potential $\phi$ which satisfies $\delta:=$ $\int_{\mathbb{R}^{d}}\left(1-\mathrm{e}^{-\phi(x)}\right) z d x<1$, we also prove that the generator $H$ has a spectral gap $\geqslant 1-\delta$. Furthermore, for any pure Gibbs state $\mu$, we derive a Poincaré inequality. The results about the spectral gap and the Poincaré inequality are a generalization and a refinement of a recent result from [Ann. Inst. H. Poincaré Probab. Statist. 38 (2002) 91-108].
\end{abstract}

(c) 2004 Elsevier SAS. All rights reserved.

\section{Résumé}

L'article est consacré à la construction et à l'étude d'une dynamique de Glauber pour les systèmes à une infinité de particules continus. Cette dynamique est un cas particulier dans la classe des processus de vie et de mort spatiaux. On considère la forme de Dirichlet associée au générateur et le processus de Markov associé. Pour un potentiel positif $\phi$ tel que $\geqslant 1-\delta$, si $\delta:=\int_{\mathbb{R}^{d}}\left(1-\mathrm{e}^{-\phi(x)}\right) z d x<1$, on montre que le générateur possède un trou spectral supérieur à $1-\delta$. Ces résultats généralisent ceux récemment obtenus dans [Ann. Inst. H. Poincaré Probab. Statist. 38 (2002) 91-108].

(c) 2004 Elsevier SAS. All rights reserved.

MSC: $60 \mathrm{~K} 35 ; 60 \mathrm{~J} 75 ; 60 \mathrm{~J} 80 ; 82 \mathrm{C} 21 ; 82 \mathrm{C} 22$

Keywords: Birth and death process; Continuous system; Gibbs measure; Glauber dynamics; Spectral gap

\footnotetext{
* Corresponding author.

E-mail addresses: kondrat@mathematik.uni-bielefeld.de (Y. Kondratiev), e.lytvynov@ swansea.ac.uk (E. Lytvynov).
} 


\section{Introduction}

This paper is devoted to the construction and study of an equilibrium Glauber-type dynamics (GD) of infinite continuous particle systems. This dynamics is a special case of a spatial birth and death process on $\mathbb{R}^{d}$. For a system of particles in a bounded volume, such processes were introduced and studied by C. Preston in [17]. In the latter case, the total number of particles is finite at any moment of time.

In the recent paper by Bertini et al., [6], the generator of the GD in a finite volume was studied. This generator corresponds to a special case of birth and death coefficients in Preston's dynamics. A positive, finite range, pair potential $\phi$ and an activity $z>0$ were fixed which satisfy the condition of the low activity-high temperature regime. Then, with any finite volume $\Lambda \subset \mathbb{R}^{d}$ and a boundary condition $\eta$ outside $\Lambda$, one may associate a finite volume Gibbs measure $\mu_{\Lambda, \eta}$. A non-local Dirichlet form $\mathcal{E}_{\Lambda, \eta}$ on $L^{2}\left(\mu_{\Lambda, \eta}\right)$ was considered which corresponds to the generator of the GD on $\Lambda$. It was shown that the generator $H_{\Lambda, \eta}$ of $\mathcal{E}_{\Lambda, \eta}$ has a spectral gap which is uniformly positive with respect to all finite volumes $\Lambda$ and boundary conditions $\eta$.

In this paper, we discuss the GD in the infinite volume. The problem of construction of a spatial birth and death process in the infinite volume was initiated in paper [8], where it was solved in a very special case of nearest neighbor birth and death processes on the real line.

So, we consider the space $\Gamma$ of all locally finite subsets (configurations) in $\mathbb{R}^{d}$, and a grand canonical Gibbs measure $\mu$ on $\Gamma$ which corresponds to a pair potential $\phi$ and activity $z>0$. The measure $\mu$ is supposed to be either of the Ruelle type or corresponding to a positive potential $\phi$ satisfying the integrability condition. In Section 2 , we shortly recall some facts about Gibbs measures which we use later on.

In Section 3, we consider the following bilinear form on $L^{2}(\Gamma, \mu)$ which is defined on a proper set of cylinder functions:

$$
\mathcal{E}(F, G)=\int_{\Gamma} \sum_{x \in \gamma}(F(\gamma \backslash x)-F(\gamma))(G(\gamma \backslash x)-G(\gamma)) \mu(d \gamma)
$$

(here and below, for simplicity of notations we will just write $x$ instead of $\{x\}$ for any $x \in \mathbb{R}^{d}$ ). We prove that this form is closable and its closure is a Dirichlet form. By using the general theory of Dirichlet forms (cf. [14]), we prove that there exists a Hunt process $\mathbf{M}$ on $\Gamma$ properly associated with $\mathcal{E}$. In particular, $\mathbf{M}$ is a conservative Markov process on $\Gamma$ with cadlag paths. By construction, $\mathbf{M}$ is an equilibrium GD on $\Gamma$ with the stationary measure $\mu$. Let us mention that the birth and death coefficients were supposed to be bounded in [8], which is not the case for the GD, provided the potential $\phi$ has a negative part.

In the case where the interaction between the particles is absent (i.e., $\phi=0$ and, therefore, $\mu$ is the Poisson measure $\pi_{z}$ with intensity $z$ ), the Markov process corresponding to the Dirichlet form (1.1) was explicitly constructed and studied by D. Surgailis [22,23].

In Sections 4 and 5, we only consider the case of a positive potential $\phi$ and study the problem of the spectral gap for the generator $H$ of the Dirichlet form $\mathcal{E}$.

Let us recall that the Poisson measure $\pi_{z}$ possesses the chaos decomposition property, and hence the space $L^{2}\left(\Gamma, \pi_{z}\right)$ is unitarily isomorphic to the symmetric Fock space over $L^{2}\left(\mathbb{R}^{d}, z d x\right)$, see e.g. [22]. Under this isomorphism, the operator $H$ goes over into the number operator $N$ in the Fock space, see [1, Theorem 5.1]. Evidently, $N$ (and thus $H$ ) has spectral gap 1. Therefore, one may expect that, at least in the case of a "small perturbation" of the Poisson measure, the operator $H$ still has a spectral gap.

One way to prove the existence of a spectral gap of a generator $H_{E}$ of a Dirichlet form $E$ is to derive a coercivity identity for $H_{E}$ on a class $\mathcal{C}$ of "nice functions," and using it, to show that, for each $F \in \mathcal{C},\left\|H_{E} F\right\|^{2} \geqslant G\left(H_{E} F, F\right)$ with $G>0$. If one additionally knows that the operator $H_{E}$ is essentially selfadjoint on $\mathcal{C}$, the latter estimate implies that $H_{E}$ has a spectral gap $\geqslant G$. In the case of a probability measure defined on a Hilbert space, this approach was developed in [10], see also [4, Ch. 6, Sect. 4].

So, having in mind this idea, we first prove in Section 4 that the operator $H$ is essentially selfadjoint. This is technically the most difficult part of the paper. Then, in Section 5, we prove a coercivity identity for the operator $H$ 
on cylinder functions, and using it and the essential selfadjointness of $H$, we show that the set $(0,1-\delta)$ does not belong to the spectrum of $H$, provided that $\delta:=\int_{\mathbb{R}^{d}}\left(1-\mathrm{e}^{-\phi(x)}\right) z d x<1$. This statement leads us to the Poincaré inequality if we are able to show that zero is a non-degenerate eigenvalue of $H$. We prove the latter statement for any $\mu$ that is an extreme point in the set of all Gibbs measures corresponding to $\phi$ and $z$. In the low activity-high temperature regime, the latter set consists of exactly one point, which is therefore extreme.

Thus, compared with the result of [6], the progress achieved in the study of the spectral gap is as follows:

1. We work in the whole space $\mathbb{R}^{d}$, instead of taking finite volumes $\Lambda$ in $\mathbb{R}^{d}$ and boundary conditions $\eta$;

2. We do not suppose that the potential $\phi$ has a finite range;

3. The essential selfadjointness of $H$ is proven;

4. For $\delta<1$, an explicit estimate for the value of the spectral gap of $H$ is found, and a Poincare inequality is proven for any pure Gibbs state.

Let us also mention the following relations between the finite volume Poincaré inequality, like in [6], and our result on the infinite volume spectral gap.

Suppose that one knows that, for any finite volume $\Lambda$ and any boundary condition $\eta$ outside $\Lambda$, the Dirichlet form $\mathcal{E}_{\Lambda, \eta}$ of the GD on $\Lambda$ satisfies the Poincare inequality with a constant $G>0$ which is independent of $\Lambda$ and $\eta$. Further suppose that an infinite volume Gibbs measure $\mu$ is a limit of a sequence of finite volume Gibbs measures $\left\{\mu_{\Lambda_{n}, \eta_{n}}, n \in \mathbb{N}\right\}$ in the weak local sense, i.e., $\int F d \mu_{\Lambda_{n}, \eta_{n}} \rightarrow \int F d \mu$ for any bounded local function $F$ on $\Gamma$. Then, one can easily derive from here that also the Dirichlet form $\mathcal{E}$ of the GD on $\mathbb{R}^{d}$ corresponding to the measure $\mu$ satisfies the Poincaré inequality with the constant $G$. In the low activity-high temperature regime, i.e., when $\delta<\exp (-1)$, the unique infinite volume Gibbs measure $\mu$ corresponding to a positive potential is the limit of finite volume Gibbs measures with empty boundary condition. Therefore, in this case, the result of [6] implies the infinite volume Poincaré inequality with a constant $G>0$. However, our result on the spectral gap of the generator and on the Poincaré inequality for any pure Gibbs state $\mu$ also holds for $\delta \in[\exp (-1), 1)$, in which case one does not yet know whether the Gibbs measure is unique and, if this is not the case, whether any pure Gibbs state is a limit of finite volume Gibbs measures.

On the other hand, although our results do not directly imply the Poincaré inequality for finite volume Gibbs measures, our proof of the spectral gap can be trivially modified to a proof of the spectral gap for finite volume Gibbs measures, again with the same estimate of the spectral gap $G \geqslant 1-\delta$. Furthermore, one can easily show that, in the finite volume case, zero is always a non-degenerate eigenvalue, so that the Poincaré inequality does also hold.

In a forthcoming paper, we are going to discuss the existence problem for general birth and death processes on configuration spaces and study a scaling limit of these processes.

\section{Gibbs measures on configuration spaces}

The configuration space $\Gamma:=\Gamma_{\mathbb{R}^{d}}$ over $\mathbb{R}^{d}, d \in \mathbb{N}$, is defined as the set of all subsets of $\mathbb{R}^{d}$ which are locally finite:

$$
\Gamma:=\left\{\gamma \subset \mathbb{R}^{d}|| \gamma_{\Lambda} \mid<\infty \text { for each compact } \Lambda \subset \mathbb{R}^{d}\right\},
$$

where $|\cdot|$ denotes the cardinality of a set and $\gamma_{\Lambda}:=\gamma \cap \Lambda$. One can identify any $\gamma \in \Gamma$ with the positive Radon measure $\sum_{x \in \gamma} \varepsilon_{x} \in \mathcal{M}\left(\mathbb{R}^{d}\right)$, where $\varepsilon_{x}$ is the Dirac measure with mass at $x, \sum_{x \in \emptyset} \varepsilon_{x}:=$ zero measure, and $\mathcal{M}\left(\mathbb{R}^{d}\right)$ stands for the set of all positive Radon measures on the Borel $\sigma$-algebra $\mathcal{B}\left(\mathbb{R}^{d}\right)$. The space $\Gamma$ can be endowed with the relative topology as a subset of the space $\mathcal{M}\left(\mathbb{R}^{d}\right)$ with the vague topology, i.e., the weakest topology on $\Gamma$ with respect to which all maps $\Gamma \ni \gamma \mapsto\langle f, \gamma\rangle:=\int_{\mathbb{R}^{d}} f(x) \gamma(d x)=\sum_{x \in \gamma} f(x), f \in \mathcal{D}$, are continuous. Here, 
$\mathcal{D}:=C_{0}\left(\mathbb{R}^{d}\right)$ is the space of all continuous real-valued functions on $\mathbb{R}^{d}$ with compact support. We will denote by $\mathcal{B}(\Gamma)$ the Borel $\sigma$-algebra on $\Gamma$.

Now, we proceed to consider Gibbs measures on $\Gamma$. A pair potential is a Borel measurable function $\phi: \mathbb{R}^{d} \rightarrow$ $\mathbb{R} \cup\{+\infty\}$ such that $\phi(-x)=\phi(x) \in \mathbb{R}$ for all $x \in \mathbb{R}^{d} \backslash\{0\}$. A grand canonical Gibbs measure $\mu$ (or just Gibbs measure for short) corresponding to the pair potential $\phi$ and activity $z>0$ is usually defined through the Dobrushin-Lanford-Ruelle equation, see e.g. [20]. However, it is convenient for us to give an equivalent definition through the Georgii-Nguyen-Zessin identity ([16, Theorem 2], see also [12, Theorem 2.2.4]).

For $\gamma \in \Gamma$ and $x \in \mathbb{R}^{d} \backslash \gamma$, we define a relative energy of interaction between a particle located at $x$ and the configuration $\gamma$ as follows:

$$
E(x, \gamma):= \begin{cases}\sum_{y \in \gamma} \phi(x-y), & \text { if } \sum_{y \in \gamma}|\phi(x-y)|<\infty, \\ +\infty, & \text { otherwise. }\end{cases}
$$

A probability measure $\mu$ on $(\Gamma, \mathcal{B}(\Gamma))$ is called a Gibbs measure if it satisfies

$$
\int_{\Gamma} \mu(d \gamma) \int_{\mathbb{R}^{d}} \gamma(d x) F(\gamma, x)=\int_{\Gamma} \mu(d \gamma) \int_{\mathbb{R}^{d}} z d x \exp [-E(x, \gamma)] F(\gamma \cup x, x)
$$

for any measurable function $F: \Gamma \times \mathbb{R}^{d} \rightarrow[0,+\infty]$. (Notice that any fixed set $\gamma \in \Gamma$ has zero Lebesgue measure, so that the expression $E(x, \gamma)$ on the right hand side of (2.1) is a.s. well-defined.) Let $\mathcal{G}(z, \phi)$ denote the set of all Gibbs measures corresponding to $z$ and $\phi$. In particular, if $\phi \equiv 0$, then (2.1) is the Mecke identity, which holds if and only if $\mu$ is the Poisson measure $\pi_{z}$ with intensity measure $z d x$.

Let us now describe some classes of Gibbs measures which appear in classical statistical mechanics of continuous systems. For every $r=\left(r^{1}, \ldots, r^{d}\right) \in \mathbb{Z}^{d}$, we define a cube

$$
Q_{r}:=\left\{x \in \mathbb{R}^{d}: r^{i}-\frac{1}{2} \leqslant x^{i}<r^{i}+\frac{1}{2}\right\} .
$$

These cubes form a partition of $\mathbb{R}^{d}$. For any $\gamma \in \Gamma$, we set $\gamma_{r}:=\gamma_{Q_{r}}, r \in \mathbb{Z}^{d}$. For $N \in \mathbb{N}$ let $\Lambda_{N}$ be the cube with side length $2 N-1$ centered at the origin in $\mathbb{R}^{d}, \Lambda_{N}$ is then a union of $(2 N-1)^{d}$ unit cubes of the form $Q_{r}$.

For $\Lambda \subset \mathbb{R}^{d}$, we denote $\Gamma_{\Lambda}:=\{\gamma \in \Gamma \mid \gamma \subset \Lambda\}$. Now, we recall some standard conditions on $\phi$.

(SS) (Superstability) There exist $A>0, B \geqslant 0$ such that, if $\gamma \in \Gamma_{\Lambda_{N}}$ for some $N$, then

$$
\sum_{\{x, y\} \subset \gamma} \phi(x-y) \geqslant \sum_{r \in \mathbb{Z}^{d}}\left(A\left|\gamma_{r}\right|^{2}-B\left|\gamma_{r}\right|\right) .
$$

Notice that the superstability condition automatically implies that the potential $\phi$ is semi-bounded from below.

(LR) (Lower regularity) There exists a decreasing positive function $a: \mathbb{N} \rightarrow \mathbb{R}_{+}$such that

$$
\sum_{r \in \mathbb{Z}^{d}} a(\|r\|)<\infty
$$

and for any $\Lambda^{\prime}, \Lambda^{\prime \prime}$ which are finite unions of cubes $Q_{r}$ and disjoint, with $\gamma^{\prime} \in \Gamma_{\Lambda^{\prime}}, \gamma^{\prime \prime} \in \Gamma_{\Lambda^{\prime \prime}}$,

$$
\sum_{x \in \gamma^{\prime}, y \in \gamma^{\prime \prime}} \phi(x-y) \geqslant-\sum_{r^{\prime}, r^{\prime \prime} \in \mathbb{Z}^{d}} a\left(\left\|r^{\prime}-r^{\prime \prime}\right\|\right)\left|\gamma_{r^{\prime}}^{\prime} \| \gamma_{r^{\prime \prime}}^{\prime \prime}\right| .
$$

Here, $\|\cdot\|$ denotes the maximum norm on $\mathbb{R}^{d}$.

(I) (Integrability)

$$
\int_{\mathbb{R}^{d}}|1-\exp [-\phi(x)]| d x<+\infty .
$$


For results related to spectral properties of the generator of the GD, we will need the following condition.

(P) (Positivity) $\phi(x) \geqslant 0$ for all $x \in \mathbb{R}^{d}$.

A probability measure $\mu$ on $(\Gamma, \mathcal{B}(\Gamma))$ is called tempered if $\mu$ is supported by

$$
S_{\infty}:=\bigcup_{n=1}^{\infty} S_{n},
$$

where

$$
S_{n}:=\left\{\gamma \in \Gamma: \forall N \in \mathbb{N} \sum_{r \in \Lambda_{N} \cap \mathbb{Z}^{d}}\left|\gamma_{r}\right|^{2} \leqslant n^{2}\left|\Lambda_{N} \cap \mathbb{Z}^{d}\right|\right\} .
$$

By $\mathcal{G}^{t}(z, \phi) \subset \mathcal{G}(z, \phi)$ we denote the set of all tempered grand canonical Gibbs measures (Ruelle measures for short). Due to [21] the set $\mathcal{G}^{t}(z, \phi)$ is non-empty for all $z>0$ and any potential $\phi$ satisfying conditions (SS), (LR), and (I). Furthermore, the set $\mathcal{G}(z, \phi)$ is non-empty for all $z>0$ and any potential $\phi$ satisfying $(\mathrm{P})$ and (I), see [12, Proposition 2.7.15].

Let us now recall the so-called Ruelle bound (cf. [21]).

Proposition 2.1. Suppose that either conditions (I), (SS), (LR) are satisfied and $\mu \in \mathcal{G}^{t}(z, \phi), z>0$, or conditions (P), (I) are satisfied and $\mu \in \mathcal{G}(z, \phi), z>0$. Then, for any $n \in \mathbb{N}$, there exists a non-negative measurable symmetric function $k_{\mu}^{(n)}$ on $\left(\mathbb{R}^{d}\right)^{n}$ such that, for any measurable symmetric function $f^{(n)}:\left(\mathbb{R}^{d}\right)^{n} \rightarrow[0, \infty]$,

$$
\int_{\Gamma} \sum_{\left\{x_{1}, \ldots, x_{n}\right\} \subset \gamma} f^{(n)}\left(x_{1}, \ldots, x_{n}\right) \mu(d \gamma)=\frac{1}{n !} \int_{\left(\mathbb{R}^{d}\right)^{n}} f^{(n)}\left(x_{1}, \ldots, x_{n}\right) k_{\mu}^{(n)}\left(x_{1}, \ldots, x_{n}\right) d x_{1} \ldots d x_{n},
$$

and

$$
\forall\left(x_{1}, \ldots, x_{n}\right) \in\left(\mathbb{R}^{d}\right)^{n}: \quad k_{\mu}^{(n)}\left(x_{1}, \ldots, x_{n}\right) \leqslant \xi^{n},
$$

where $\xi>0$ is independent of $n$.

The functions $k_{\mu}^{(n)}, n \in \mathbb{N}$, are called correlation functions of the measure $\mu$, while (2.3) is called the Ruelle bound.

Notice that any measure $\mu \in \mathcal{G}(z, \phi)$ as in Proposition 2.1 satisfies

$$
\int_{\Gamma}\langle\varphi, \gamma\rangle^{n} \mu(d \gamma)<\infty, \quad \varphi \in \mathcal{D}, \varphi \geqslant 0, n \in \mathbb{N},
$$

that is, $\mu$ has all local moments finite.

\section{The Dirichlet form $\mathcal{E}$ and associated Markov process}

We introduce a set $\mathcal{F} C_{\mathrm{b}}(\mathcal{D}, \Gamma)$ of all functions on $\Gamma$ of the form

$$
F(\gamma)=g_{F}\left(\left\langle\varphi_{1}, \gamma\right\rangle, \ldots,\left\langle\varphi_{N}, \gamma\right\rangle\right),
$$

where $N \in \mathbb{N}, \varphi_{1}, \ldots, \varphi_{N} \in \mathcal{D}$, and $g_{F} \in C_{\mathrm{b}}\left(\mathbb{R}^{N}\right)$. Here, $C_{\mathrm{b}}\left(\mathbb{R}^{N}\right)$ denotes the set of all continuous bounded functions on $\mathbb{R}^{N}$. For any $\gamma \in \Gamma$, we consider $T_{\gamma}:=L^{2}\left(\mathbb{R}^{d}, \gamma\right)$ as a "tangent" space to $\Gamma$ at the point $\gamma$, and for any $F \in \mathcal{F} C_{\mathrm{b}}(\mathcal{D}, \Gamma)$ we define the "gradient" of $F$ at $\gamma$ as the element of $T_{\gamma}$ given by 


$$
D^{-} F(\gamma, x):=D_{x}^{-} F(\gamma):=F(\gamma \backslash x)-F(\gamma), \quad x \in \mathbb{R}^{d} .
$$

(Evidently, $D^{-} F(\gamma)$ indeed belongs to $T_{\gamma}$.)

Let $\mu$ be a Gibbs measure as in Proposition 2.1. We will preserve the notation $\mathcal{F} C_{\mathrm{b}}(\mathcal{D}, \Gamma)$ for the set of all $\mu$-classes of functions from $\mathcal{F} C_{\mathrm{b}}(\mathcal{D}, \Gamma)$. The set $\mathcal{F} C_{\mathrm{b}}(\mathcal{D}, \Gamma)$ is dense in $L^{2}(\Gamma, \mu)$. We now define

$$
\begin{aligned}
\mathcal{E}(F, G) & :=\int_{\Gamma}\left(D^{-} F(\gamma), D^{-} G(\gamma)\right)_{T_{\gamma}} \mu(d \gamma) \\
& =\int_{\Gamma} \mu(d \gamma) \int_{\mathbb{R}^{d}} \gamma(d x) D_{x}^{-} F(\gamma) D_{x}^{-} G(\gamma), \quad F, G \in \mathcal{F} C_{\mathrm{b}}(\mathcal{D}, \Gamma) .
\end{aligned}
$$

Notice that, for any $F \in \mathcal{F} C_{\mathrm{b}}(\mathcal{D}, \Gamma)$, there exists $f \in \mathcal{D}$ such that $\left|D_{x}^{-} F(\gamma)\right| \leqslant f(x)$ for all $\gamma \in \Gamma$ and $x \in \gamma$. Hence, by (2.4), the right hand side of (3.2) is well defined. By (2.1), we also get, for $F, G \in \mathcal{F} C_{\mathrm{b}}(\mathcal{D}, \Gamma)$,

$$
\mathcal{E}(F, G)=\int_{\Gamma} \mu(d \gamma) \int_{\mathbb{R}^{d}} z d x \exp [-E(x, \gamma)] D_{x}^{+} F(\gamma) D_{x}^{+} G(\gamma),
$$

where $D_{x}^{+} F(\gamma):=F(\gamma \cup x)-F(\gamma)$.

Lemma 3.1. We have: $\mathcal{E}(F, G)=0$ for all $F, G \in \mathcal{F} C_{\mathrm{b}}(\mathcal{D}, \Gamma)$ such that $F=0$ u-a.e.

Proof. Let $F \in \mathcal{F} C_{\mathrm{b}}(\mathcal{D}, \Gamma), F=0 \mu$-a.e. Denote $B(r):=\left\{x \in \mathbb{R}^{d}:|x|<r\right\}$. Then, by (2.1),

$$
0=\int_{\Gamma} \mu(d \gamma) \int_{B(r)} \gamma(d x)|F(\gamma)|=\int_{\Gamma} \mu(d \gamma) \int_{B(r)} z d x \exp [-E(x, \gamma)]|F(\gamma \cup x)| .
$$

Hence, $F(\gamma \cup x)=0$ for $\mu(d \gamma) z d x \exp [-E(x, \gamma)]$-a.e. $(\gamma, x) \in \Gamma \times \mathbb{R}^{d}$. Therefore, by (3.3), the lemma follows.

Thus, $\left(\mathcal{E}, \mathcal{F} C_{\mathrm{b}}(\mathcal{D}, \Gamma)\right)$ is a well-defined bilinear form on $L^{2}(\Gamma, \mu)$.

Proposition 3.1. We have:

$$
\mathcal{E}(F, G)=\int_{\Gamma} H F(\gamma) G(\gamma) \mu(d \gamma), \quad F, G \in \mathcal{F} C_{\mathrm{b}}(\mathcal{D}, \Gamma),
$$

where

$$
H F(\gamma)=-\int_{\mathbb{R}^{d}} z d x \exp [-E(x, \gamma)] D_{x}^{+} F(\gamma)-\int_{\mathbb{R}^{d}} \gamma(d x) D_{x}^{-} F(\gamma)
$$

and $H F \in L^{2}(\Gamma, \mu)$. The bilinear form $\left(\mathcal{E}, \mathcal{F} C_{\mathrm{b}}(\mathcal{D}, \Gamma)\right)$ is closable on $L^{2}(\Gamma, \mu)$ and its closure will be denoted by $(\mathcal{E}, D(\mathcal{E}))$. The operator $\left(H, \mathcal{F} C_{\mathrm{b}}(\mathcal{D}, \Gamma)\right)$ in $L^{2}(\Gamma, \mu)$ has Friedrichs' extension, which we denote by $(H, D(H))$.

Proof. Eqs. (3.4), (3.5) easily follow from (2.1) and (3.2). Let us show that $H F \in L^{2}(\Gamma, \mu)$. By (2.4), the inclusion $\int_{\mathbb{R}^{d}} \gamma(d x) D_{x}^{-} F(\gamma) \in L^{2}(\Gamma, \mu)$ is trivial. Next, we can find a compact $\Lambda \subset \mathbb{R}^{d}$ and $C_{1}>0$ such that $\left|D_{x}^{+} F(\gamma)\right| \leqslant$ $C_{1} \chi_{\Lambda}(x)$ for all $\gamma \in \Gamma$ and $x \in \mathbb{R}^{d}$. Here, $\chi_{\Lambda}$ denotes the indicator of $\Lambda$. Hence, by (2.1) and (2.2), 


$$
\begin{aligned}
& \int_{\Gamma}\left(\int_{\mathbb{R}^{d}} z d x \exp [-E(x, \gamma)] D_{x}^{+} F(\gamma)\right)^{2} \mu(d \gamma) \\
& \quad \leqslant C_{1}^{2} \int_{\Gamma} \mu(d \gamma) \int_{\Lambda} z d x \int_{\Lambda} z d y \exp [-E(x, \gamma)-E(y, \gamma)-\phi(x-y)] \exp [\phi(x-y)] \\
& \quad=2 C_{1}^{2} \int_{\Gamma} \sum_{\{x, y\} \in \gamma_{\Lambda}} \exp [\phi(x-y)] \mu(d \gamma)=C_{1}^{2} \int_{\Lambda^{2}} \exp [\phi(x-y)] k_{\mu}^{(2)}(x, y) d x d y<\infty
\end{aligned}
$$

since $k_{\mu}^{(2)}(x, y) \leqslant C_{2} \exp [-\phi(x-y)]$ for all $x, y \in \mathbb{R}^{d}, C_{2}>0$, cf. [2, Eq. (4.29)]. Therefore, $H$ is the $L^{2}(\Gamma, \mu)$ generator of the bilinear form $\mathcal{E}$. The rest of the proposition now follows from e.g. [18, Theorem X.23].

For the notion of a "Dirichlet form", appearing in the following lemma, we refer to e.g. [14, Chapter I, Section 4].

Lemma 3.2. $(\mathcal{E}, D(\mathcal{E}))$ is a Dirichlet form on $L^{2}(\Gamma, \mu)$.

Proof. On $D(\mathcal{E})$ consider the norm $\|F\|_{D(\mathcal{E})}:=\left(\|F\|_{L^{2}(\mu)}^{2}+\mathcal{E}(F)\right)^{1 / 2}, F \in D(\mathcal{E})$. Here, we denoted $\mathcal{E}(F):=$ $\mathcal{E}(F, F)$. For any $F, G \in \mathcal{F} C_{\mathrm{b}}(\mathcal{D}, \Gamma)$, we define

$$
S(F, G)(x, \gamma):=D_{x}^{-} F(\gamma) D_{x}^{-} G(\gamma), \quad x \in \mathbb{R}^{d}, \gamma \in \Gamma .
$$

Using the Cauchy-Schwarz inequality, we conclude that $S$ extends to a bilinear continuous map from $(D(\mathcal{E})$, $\left.\|\cdot\|_{D(\mathcal{E})}\right) \times\left(D(\mathcal{E}),\|\cdot\|_{D(\mathcal{E})}\right)$ into $L^{1}\left(\mathbb{R}^{d} \times \Gamma, \tilde{\mu}\right)$, where $\tilde{\mu}(d x, d \gamma):=\gamma(d x) \mu(d \gamma)$. Let $F \in D(\mathcal{E})$ and consider any sequence $F_{n} \in \mathcal{F} C_{\mathrm{b}}(\mathcal{D}, \Gamma), n \in \mathbb{N}$, such that $F_{n} \rightarrow F$ in $\left(D(\mathcal{E}),\|\cdot\|_{D(\mathcal{E})}\right)$ as $n \rightarrow \infty$. Then, $F_{n}(\gamma) \rightarrow F(\gamma)$ as $n \rightarrow \infty$ for $\mu$-a.e. $\gamma \in \Gamma$ (if necessary, take a subsequence of $\left(F_{n}\right)_{n \in \mathbb{N}}$ with this property). Furthermore, for any $r>0$, we have, analogously to (3.6):

$$
\begin{aligned}
& \int_{B(r) \times \Gamma}\left|F_{n}(\gamma \backslash x)-F(\gamma \backslash x)\right| \tilde{\mu}(d x, d \gamma) \\
& \quad=\int_{\Gamma} \mu(d \gamma) \int_{B(r)} z d x \exp [-E(x, \gamma)]\left|F_{n}(\gamma)-F(\gamma)\right| \\
& \quad \leqslant\left(\int_{\Gamma}\left|F_{n}(\gamma)-F(\gamma)\right|^{2} \mu(d \gamma)\right)^{1 / 2}\left(\int\left(\int_{\Gamma} z d x \exp [-E(x, \gamma)]\right)^{2} \mu(d \gamma)\right)^{1 / 2} \rightarrow 0
\end{aligned}
$$

as $n \rightarrow \infty$. Therefore, $F_{n}(\gamma \backslash x) \rightarrow F(\gamma \backslash x)$ for $\tilde{\mu}$-a.e. $(x, \gamma) \in \mathbb{R}^{d} \times \Gamma$. Thus, $D_{x}^{-} F_{n}(\gamma) \rightarrow D_{x}^{-} F(\gamma)$ as $n \rightarrow \infty$ for $\tilde{\mu}$-a.e. $(x, \gamma) \in \mathbb{R}^{d} \times \Gamma$, which yields:

$$
S(F, G)(x, \gamma)=D_{x}^{-} F(\gamma) D_{x}^{-} G(\gamma), \quad \tilde{\mu} \text {-a.e. }(x, \gamma) \in \mathbb{R}^{d} \times \Gamma, F, G \in D(\mathcal{E}) .
$$

Hence,

$$
\mathcal{E}(F, G)=\int_{\mathbb{R}^{d} \times \Gamma} D_{x}^{-} F(\gamma) D_{x}^{-} G(\gamma) \tilde{\mu}(d x, d \gamma), \quad F, G \in D(\mathcal{E}) .
$$

Define $\mathbb{R} \ni x \mapsto g(x):=(0 \vee x) \wedge 1$. We again fix any $F \in D(\mathcal{E})$ and let $\left(F_{n}\right)_{n \in \mathbb{N}}$ be a sequence of functions from $\mathcal{F} C_{\mathrm{b}}(\mathcal{D}, \Gamma)$ such that $F_{n} \rightarrow F$ in $\left(D(\mathcal{E}),\|\cdot\|_{D(\mathcal{E})}\right)$. Consider the sequence $\left(g\left(F_{n}\right)\right)_{n \in \mathbb{N}}$. We evidently have: $g\left(F_{n}\right) \in$ $\mathcal{F} C_{\mathrm{b}}(\mathcal{D}, \Gamma)$ for each $n \in \mathbb{N}$ and $g\left(F_{n}\right) \rightarrow g(F)$ as $n \rightarrow \infty$ in $L^{2}(\Gamma, \mu)$. Next, by the above argument, we have: 
$D_{x}^{-} g\left(F_{n}(\gamma)\right) \rightarrow D_{x}^{-} g(F(\gamma))$ as $n \rightarrow \infty$ for $\tilde{\mu}$-a.e. $(x, \gamma)$. Furthermore, the sequence $\left(D_{x}^{-} g\left(F_{n}(\gamma)\right)\right)_{n \in \mathbb{N}}$ is $\tilde{\mu}$ uniformly square-integrable, since so is the sequence $\left(D_{x}^{-} F_{n}(\gamma)\right)_{n \in \mathbb{N}}$. Therefore, the sequence $\left(D_{x}^{-} g\left(F_{n}(\gamma)\right)\right)_{n \in \mathbb{N}}$ converges to $D_{x}^{-} g(F(\gamma))$ in $L^{2}\left(\mathbb{R}^{d} \times \Gamma, \tilde{\mu}\right)$. This yields: $g(F) \in D(\mathcal{E})$.

For any $x, y \in \mathbb{R}$, we evidently have $|g(x)-g(y)| \leqslant|x-y|$. By (3.9), we then finally have: $\mathcal{E}(g(F)) \leqslant \mathcal{E}(F)$, which means that $(\mathcal{E}, D(\mathcal{E}))$ is a Dirichlet form.

We will now need the bigger space $\ddot{\Gamma}$ consisting of all $\mathbb{Z}_{+}$-valued Radon measures on $\mathbb{R}^{d}$ (which is Polish, see e.g. [9]). Since $\Gamma \subset \ddot{\Gamma}$ and $\mathcal{B}(\ddot{\Gamma}) \cap \Gamma=\mathcal{B}(\Gamma)$, we can consider $\mu$ as a measure on $(\ddot{\Gamma}, \mathcal{B}(\ddot{\Gamma}))$ and correspondingly $(\mathcal{E}, D(\mathcal{E}))$ as a Dirichlet form on $L^{2}(\ddot{\Gamma}, \mu)$.

For the notion of a "quasi-regular Dirichlet form", appearing in the following lemma, we refer to [14, Chapter IV, Section 3].

Proposition 3.2. $(\mathcal{E}, D(\mathcal{E}))$ is a quasi-regular Dirichlet form on $L^{2}(\ddot{\Gamma}, \mu)$.

Proof. By [15, Proposition 4.1], it suffices to show that there exists a bounded, complete metric $\rho$ on $\ddot{\Gamma}$ generating the vague topology such that, for all $\gamma_{0} \in \ddot{\Gamma}, \rho\left(\cdot, \gamma_{0}\right) \in D(\mathcal{E})$ and $\int_{\mathbb{R}^{d}} S\left(\rho\left(\cdot, \gamma_{0}\right)\right)(x, \gamma) \gamma(d x) \leqslant \eta(\gamma) \mu$-a.e. for some $\eta \in L^{1}(\ddot{\Gamma}, \mu)$ (independent of $\left.\gamma_{0}\right)$. Here, $S(F):=S(F, F)$. The proof below is a modification of the proof of [15, Proposition 4.8].

For each $k \in \mathbb{N}$, we define

$$
g_{k}(x):=\frac{2}{3}\left(\frac{1}{2}-d(x, B(k)) \wedge \frac{1}{2}\right), \quad x \in \mathbb{R}^{d},
$$

where $d(x, B(k))$ denotes the distance from the point $x$ to the open ball $B(k)$. Next, we set

$$
\phi_{k}(x):=3 g_{k}(x), \quad x \in \mathbb{R}^{d}, k \in \mathbb{N} .
$$

Let $\zeta$ be a function from $C_{\mathrm{b}}^{1}(\mathbb{R})$ such that $0 \leqslant \zeta \leqslant 1$ on $[0, \infty), \zeta(t)=t$ on $[-1 / 2,1 / 2], \zeta^{\prime} \in[0,1]$ on $[0, \infty)$. For any fixed $\gamma_{0} \in \ddot{\Gamma}$ and for any $k, n \in \mathbb{N}$, (the restriction to $\Gamma$ of) the function

$$
\zeta\left(\sup _{j \leqslant n}\left|\left\langle\phi_{k} g_{j}, \cdot\right\rangle-\left\langle\phi_{k} g_{j}, \gamma_{0}\right\rangle\right|\right)
$$

belongs to $\mathcal{F} C_{\mathrm{b}}(\mathcal{D}, \Gamma)$ (note that $\left\langle\phi_{k} g_{j}, \gamma_{0}\right\rangle$ is a constant). Furthermore, taking to notice that $\zeta^{\prime} \in[0,1]$ on $[0, \infty)$, we get from the mean value theorem

$$
\begin{aligned}
& S\left(\zeta\left(\sup _{j \leqslant n}\left|\left\langle\phi_{k} g_{j}, \cdot\right\rangle-\left\langle\phi_{k} g_{j}, \gamma_{0}\right\rangle\right|\right)\right)(x, \gamma) \\
& \quad \leqslant\left(\sup _{j \leqslant n}\left|\left\langle\phi_{k} g_{j}, \gamma\right\rangle-\left\langle\phi_{k} g_{j}, \gamma_{0}\right\rangle-\phi_{k}(x) g_{j}(x)\right|-\sup _{j \leqslant n}\left|\left\langle\phi_{k} g_{j}, \gamma\right\rangle-\left\langle\phi_{k} g_{j}, \gamma_{0}\right\rangle\right|\right)^{2} \\
& \quad \leqslant \sup _{j \leqslant n} \phi_{k}^{2}(x) g_{j}^{2}(x) \leqslant \chi_{B(k+1 / 2)}(x) .
\end{aligned}
$$

Set

$$
c_{k}:=\left(1+\int_{B(k+1 / 2)} k_{\mu}^{(1)}(x) d x\right)^{-1 / 2} 2^{-k / 2} .
$$

Using estimate (3.10) and the numbers $c_{k}$, we now easily obtain the statement of the proposition absolutely analogously to the proof of [15, Lemma 4.11 and Proposition 4.8].

For the notion of an " $\mathcal{E}$-exceptional set", appearing in the next proposition, we refer to e.g. [14, Chapter III, Section 2]. 


\section{Proposition 3.3. The set $\ddot{\Gamma} \backslash \Gamma$ is $\mathcal{E}$-exceptional.}

Proof. We modify the proof of [19, Proposition 1 and Corollary 1] according to our situation.

It suffices to prove the result locally, that is, to show that, for every fixed $a \in \mathbb{N}$, the set

$$
N:=\left\{\gamma \in \ddot{\Gamma}: \sup \left(\gamma(\{x\}): x \in[-a, a]^{d}\right) \geqslant 2\right\}
$$

is $\mathcal{E}$-exceptional. By [19, Lemma 1], we need to prove that there exists a sequence $u_{n} \in D(\mathcal{E}), n \in \mathbb{N}$, such that each $u_{n}$ is a continuous function on $\ddot{\Gamma}, u_{n} \rightarrow \chi_{N}$ pointwise as $n \rightarrow \infty$, and $\sup _{n \in \mathbb{N}} \mathcal{E}\left(u_{n}\right)<\infty$.

Let $f \in C_{0}(\mathbb{R})$ be such that $\chi_{[0,1]} \leqslant f \leqslant \chi_{[-1 / 2,3 / 2)}$. For any $n \in \mathbb{N}$ and $i=\left(i_{1}, \ldots, i_{d}\right) \in \mathbb{Z}^{d}$, define a function $f_{i}^{(n)} \in \mathcal{D}$ by

$$
f_{i}^{(n)}(x):=\prod_{k=1}^{d} f\left(n x_{k}-i_{k}\right), \quad x \in \mathbb{R}^{d} .
$$

Let also

$$
I_{i}^{(n)}(x):=\prod_{k=1}^{d} \chi_{[-1 / 2,3 / 2)}\left(n x_{k}-i_{k}\right), \quad x \in \mathbb{R}^{d},
$$

and note that $f_{i}^{(n)} \leqslant I_{i}^{(n)}$.

Let $\psi \in C_{\mathrm{b}}^{1}(\mathbb{R})$ be such that $\chi_{[2, \infty)} \leqslant \psi \leqslant \chi_{[1, \infty)}$ and $0 \leqslant \psi^{\prime} \leqslant 2 \chi_{(1, \infty)}$. Set $\mathcal{A}_{n}:=\mathbb{Z}^{d} \cap[-n a, n a]^{d}$ and define continuous functions

$$
\ddot{\Gamma} \ni \gamma \mapsto u_{n}(\gamma):=\psi\left(\sup _{i \in \mathcal{A}_{n}}\left\langle f_{i}^{(n)}, \gamma\right\rangle\right), \quad n \in \mathbb{N},
$$

whose restriction to $\Gamma$ belongs to $\mathcal{F} C_{\mathrm{b}}(\mathcal{D}, \Gamma)$. Evidently, $u_{n} \rightarrow \chi_{N}$ pointwise as $n \rightarrow \infty$. We now have:

$$
S\left(u_{n}\right)(x, \gamma)=\left(\psi\left(\sup _{i \in \mathcal{A}_{n}}\left\langle f_{i}^{(n)}, \gamma-\varepsilon_{x}\right\rangle\right)-\psi\left(\sup _{i \in \mathcal{A}_{n}}\left\langle f_{i}^{(n)}, \gamma\right\rangle\right)\right)^{2} \quad \tilde{\mu} \text {-a.e. }
$$

and by the mean value theorem, we get, for $\tilde{\mu}$-a.e. $(x, \gamma) \in \mathbb{R}^{d} \times \ddot{\Gamma}$,

$$
S\left(u_{n}\right)(x, \gamma)=\psi^{\prime}\left(T_{n}(\gamma, x)\right)^{2}\left(\sup _{i \in \mathcal{A}_{n}}\left\langle f_{i}^{(n)}, \gamma-\varepsilon_{x}\right\rangle-\sup _{i \in \mathcal{A}_{n}}\left\langle f_{i}^{(n)}, \gamma\right\rangle\right)^{2},
$$

where $T_{n}(\gamma, x) \in \mathbb{R}$ is a point between $\sup _{i \in \mathcal{A}_{n}}\left\langle f_{i}^{(n)}, \gamma-\varepsilon_{x}\right\rangle$ and $\sup _{i \in \mathcal{A}_{n}}\left\langle f_{i}^{(n)}, \gamma\right\rangle$. Next, for any $\gamma \in \ddot{\Gamma}$ and $x \in \mathbb{R}^{d}$ :

$$
\begin{aligned}
\left|\sup _{i \in \mathcal{A}_{n}}\left\langle f_{i}^{(n)}, \gamma-\varepsilon_{x}\right\rangle-\sup _{i \in \mathcal{A}_{n}}\left\langle f_{i}^{(n)}, \gamma\right\rangle\right| & \leqslant \sup _{i \in \mathcal{A}_{n}}\left|\left\langle f_{i}^{(n)}, \gamma-\varepsilon_{x}\right\rangle-\left\langle f_{i}^{(n)}, \gamma\right\rangle\right| \\
& =\sup _{i \in \mathcal{A}_{n}} f_{i}^{(n)}(x) \leqslant \sup _{i \in \mathcal{A}_{n}} I_{i}^{(n)}(x) \leqslant \chi_{[-a-1, a+1]^{d}}(x) .
\end{aligned}
$$

We evidently have, for each $\gamma \in \ddot{\Gamma}$ and $x \in \operatorname{supp}(\gamma)$ :

$$
\sup _{i \in \mathcal{A}_{n}}\left\langle f_{i}^{(n)}, \gamma-\varepsilon_{x}\right\rangle \leqslant T_{n}(\gamma, x) \leqslant \sup _{i \in \mathcal{A}_{n}}\left\langle f_{i}^{(n)}, \gamma\right\rangle .
$$

Hence,

$$
\psi^{\prime}\left(T_{n}(\gamma, x)\right)^{2} \leqslant 4 \chi_{\left\{\sup _{i \in \mathcal{A}_{n}}\left\langle f_{i}^{(n)}, \cdot\right\rangle>1\right\}}(\gamma) \leqslant 4 \chi_{\left\{\sup _{i \in \mathcal{A}_{n}}\left\langle I_{i}^{(n)}, \cdot\right\rangle \geqslant 2\right\}}(\gamma) \leqslant 4 \sum_{i \in \mathcal{A}_{n}} \chi_{\left\{\left\langle I_{i}^{(n)}, \cdot\right\rangle \geqslant 2\right\}}(\gamma),
$$


where we used the fact that $I_{i}^{(n)}$ is integer-valued. By (3.11)-(3.13), we have, for $\tilde{\mu}$-a.e. $(x, \gamma) \in \mathbb{R}^{d} \times \ddot{\Gamma}$ :

$$
S\left(u_{n}\right)(x, \gamma) \leqslant 4 \sum_{i \in \mathcal{A}_{n}} \chi_{\left\{\left\langle I_{i}^{(n)}, \cdot\right\rangle \geqslant 2\right\}}(\gamma) \chi_{[-a-1, a+1]^{d}}(x) .
$$

Therefore, by the Cauchy-Schwarz inequality and (3.9),

$$
\mathcal{E}\left(u_{n}\right) \leqslant 4 \sum_{i \in \mathcal{A}_{n}}\left(\mu\left(\left\{\left\langle I_{i}^{(n)}, \cdot\right\rangle \geqslant 2\right\}\right)\right)^{1 / 2}\left(\int_{\ddot{\Gamma}}\left\langle\gamma, \chi_{[-a-1, a+1]^{d}}\right\rangle \mu(d \gamma)\right)^{1 / 2} .
$$

By using [21, Theorem 5.5], we easily conclude that there exists a constant $C_{3}>0$, independent of $i$ and $n$, such that, for all $i \in \mathcal{A}_{n}$ and $n \in \mathbb{N}$,

$$
\mu\left(\left\{\left\langle I_{i}^{(n)}, \cdot\right\rangle \geqslant 2\right\}\right) \leqslant C_{3}\left(\int_{\mathbb{R}^{d}} I_{i}^{(n)}(x) d x\right)^{2}=C_{3}\left(\frac{2}{n}\right)^{2 d} .
$$

Since $\left|\mathcal{A}_{n}\right|=(2 n a+1)^{d}$, we get from (2.2), (3.14), and (3.15):

$$
\mathcal{E}\left(u_{n}\right) \leqslant 4 C_{3}^{1 / 2}(2 n a+1)^{d}\left(\frac{2}{n}\right)^{d}\left(\int_{[-a-1, a+1]^{d}} k_{\mu}^{(1)}(x) d x\right)^{1 / 2}, \quad n \in \mathbb{N} .
$$

Therefore, there exists a constant $C_{4}>0$, independent of $n$, such that $\mathcal{E}\left(u_{n}\right) \leqslant C_{4}$ for all $n \in \mathbb{N}$.

We now have the main result of this section.

Theorem 3.1. (1) Suppose that the conditions of Proposition 2.1 are satisfied. Then, there exists a Hunt process

$$
\mathbf{M}=\left(\boldsymbol{\Omega}, \mathbf{F},\left(\mathbf{F}_{t}\right)_{t \geqslant 0},\left(\boldsymbol{\Theta}_{t}\right)_{t \geqslant 0},(\mathbf{X}(t))_{t \geqslant 0},\left(\mathbf{P}_{\gamma}\right)_{\gamma \in \Gamma}\right)
$$

on $\Gamma$ (see e.g. $[14$, p. 92]) which is properly associated with $(\mathcal{E}, D(\mathcal{E}))$, i.e., for all ( $\mu$-versions of $) F \in L^{2}(\Gamma, \mu)$ and all $t>0$ the function

$$
\Gamma \ni \gamma \mapsto p_{t} F(\gamma):=\int_{\Omega} F(\mathbf{X}(t)) d \mathbf{P}_{\gamma}
$$

is an $\mathcal{E}$-quasi-continuous version of $\exp (-t H) F$, where $H$ is the generator of $(\mathcal{E}, D(\mathcal{E}))$. $\mathbf{M}$ is up to $\mu$-equivalence unique (cf. [14, Chapter IV, Section 6]). In particular, $\mathbf{M}$ is $\mu$-symmetric (i.e., $\int G p_{t} F d \mu=\int F p_{t} G d \mu$ for all $F, G: \Gamma \rightarrow \mathbb{R}_{+}, \mathcal{B}(\Gamma)$-measurable) and has $\mu$ as an invariant measure.

(2) $\mathbf{M}$ from (1) is up to $\mu$-equivalence (cf. [14, Definition 6.3]) unique between all Hunt processes $\mathbf{M}^{\prime}=$ $\left(\boldsymbol{\Omega}^{\prime}, \mathbf{F}^{\prime},\left(\mathbf{F}_{t}^{\prime}\right)_{t \geqslant 0},\left(\boldsymbol{\Theta}_{t}^{\prime}\right)_{t \geqslant 0},\left(\mathbf{X}^{\prime}(t)\right)_{t \geqslant 0},\left(\mathbf{P}_{\gamma}^{\prime}\right)_{\gamma \in \Gamma}\right)$ on $\Gamma$ having $\mu$ as an invariant measure and solving the martingale problem for $(-H, D(H))$, i.e., for all $G \in D(H)$

$$
\widetilde{G}\left(\mathbf{X}^{\prime}(t)\right)-\widetilde{G}\left(\mathbf{X}^{\prime}(0)\right)+\int_{0}^{t}(H G)\left(\mathbf{X}^{\prime}(s)\right) d s, \quad t \geqslant 0,
$$

is an $\left(\mathbf{F}_{t}^{\prime}\right)$-martingale under $\mathbf{P}_{\gamma}^{\prime}$ for $\mathcal{E}$-q.e. $\gamma \in \Gamma$. (Here, $\widetilde{G}$ denotes a quasi-continuous version of $G$, $c f .[14$, Chapter IV, Proposition 3.3].)

Remark 3.1. In fact, the statement of Theorem 3.1 remains true for any Gibbs measure $\mu \in \mathcal{G}(z, \phi)$ whose correlation functions satisfy the Ruelle bound. 
Proof of Theorem 3.1. The first part of the theorem follows from Propositions 3.2, 3.3 and [14, Chapter IV, Theorem 3.5 and Chapter V, Proposition 2.15]. The second part follows directly from (the proof of) [3, Theorem 3.5].

In the above theorem, $\mathbf{M}$ is canonical, i.e., $\boldsymbol{\Omega}$ is the set of all cadlag functions $\omega:[0, \infty) \rightarrow \Gamma$ (i.e., $\omega$ is right continuous on $[0, \infty)$ and has left limits on $(0, \infty)), \mathbf{X}(t)(\omega):=\omega(t), t \geqslant 0, \omega \in \boldsymbol{\Omega},\left(\mathbf{F}_{t}\right)_{t \geqslant 0}$ together with $\mathbf{F}$ is the corresponding minimum completed admissible family (cf. [7, Section 4.1]) and $\boldsymbol{\Theta}_{t}, t \geqslant 0$, are the corresponding natural time shifts.

\section{Selfadjointness of the generator}

In what follows, we will always suppose that the potential $\phi$ is positive.

Theorem 4.1. Suppose that conditions (P), (I) are satisfied and $\mu \in \mathcal{G}(z, \phi), z>0$. Then, the operator $\left(H, \mathcal{F} C_{\mathrm{b}}(\mathcal{D}, \Gamma)\right)$ is essentially selfadjoint in $L^{2}(\Gamma, \mu)$. In particular, Friedrichs' extension of $\left(H, \mathcal{F} C_{\mathrm{b}}(\mathcal{D}, \Gamma)\right)$ coincides with its closure.

Proof. Let $(\tilde{H}, D(\tilde{H}))$ denote the closure of $\left(H, \mathcal{F} C_{\mathrm{b}}(\mathcal{D}, \Gamma)\right)$, which exists since the latter operator is a Hermitian one. We have to show that $(\tilde{H}, D(\tilde{H}))$ is selfadjoint. Since $\tilde{H} \geqslant 0$, by the Nussbaum theorem, it is enough to show that there exists a set $\mathcal{S} \subset \bigcap_{n=1}^{\infty} D\left(\tilde{H}^{n}\right)$ which is total in $L^{2}(\Gamma, \mu)$ and each $F \in \mathcal{S}$ satisfies:

$$
\sum_{n=0}^{\infty} \frac{\left\|\tilde{H}^{n} F\right\|_{L^{2}(\mu)}}{(2 n) !} t^{n}<\infty
$$

for some $t>0$, see e.g. [18, Theorem X.40].

Let us recall some well-known facts about cylinder functions on the configuration space (see e.g. [11] for details). Let $\mathcal{O}_{\mathrm{c}}\left(\mathbb{R}^{d}\right)$ denote the set of all open, relatively compact sets in $\mathbb{R}^{d}$. For $\Lambda \in \mathcal{O}_{\mathrm{c}}\left(\mathbb{R}^{d}\right)$, we have: $\Gamma_{\Lambda}=$ $\bigsqcup_{n=0}^{\infty} \Gamma_{\Lambda}^{(n)}$, where $\Gamma_{\Lambda}^{(n)}$ denotes the set of all $n$-point subsets of $\Lambda, n \in \mathbb{N}$, and $\Gamma_{\Lambda}^{(0)}=\{\emptyset\}$. For $n \in \mathbb{N}$, we can naturally identify $\Gamma_{\Lambda}^{(n)}$ with $\tilde{\Lambda}^{n} / S_{n}$, where $\tilde{\Lambda}^{n}:=\left\{\left(x_{1}, \ldots, x_{n}\right) \in \Lambda^{n}: x_{i} \neq x_{j}\right.$ if $\left.i \neq j\right\}$ and $S_{n}$ denotes the group of permutations of $\{1, \ldots, n\}$ that acts on $\tilde{\Lambda}^{n}$ by permuting the numbers of the coordinates. Furthermore, the trace $\sigma$-algebra of $\mathcal{B}(\Gamma)$ on $\Gamma_{\Lambda}^{(n)}$ coincides with the $\sigma$-algebra $\mathcal{B}_{\text {sym }}\left(\tilde{\Lambda}^{n}\right)$ of all symmetric Borel subsets of $\tilde{\Lambda}^{n}$ (again under a natural isomorphism). Finally, any measurable function $F_{\Lambda}$ on $\Gamma_{\Lambda}$ may be identified with a measurable cylinder function $F$ on $\Gamma$ by setting $\Gamma \ni \gamma \mapsto F(\gamma):=F_{\Lambda}\left(\gamma_{\Lambda}\right)$.

Lemma 4.1. Suppose that the conditions of Theorem 4.1 are satisfied. Let $\Lambda \in \mathcal{O}_{\mathrm{c}}\left(\mathbb{R}^{d}\right)$ and let $F$ be a measurable bounded function $F$ on $\Gamma_{\Lambda}$ such that $F\left\lceil\Gamma_{\Lambda}^{(n)} \equiv 0\right.$ for all $n \geqslant N, N \in \mathbb{N}$. Then, $F \in D(\tilde{H})$ and the action of $\tilde{H}$ on $F$ is given by the right hand side of (3.5).

Proof. We take arbitrary, open, disjoint subsets $O_{1}, \ldots, O_{n}$ of $\Lambda$. Consider functions $g_{1}, g_{2} \in C_{\mathrm{b}}(\mathbb{R})$ such that $g_{1}(1)=1, g_{2}(0)=1$ and $g_{1}(x)=0$ if $|x-1|>1 / 2, g_{2}(x)=0$ if $|x|>1 / 2$. Approximating the indicator functions $\chi_{O_{i}}, i=1, \ldots, n$, and $\chi_{\Lambda \backslash\left(O_{1} \cup \ldots \cup O_{n}\right)}$ by functions from $\mathcal{D}$, we easily conclude that the statement of the lemma holds for the function

$$
F(\gamma)=g_{1}\left(\left\langle\chi O_{1}, \gamma\right\rangle\right) \cdots g_{1}\left(\left\langle\chi O_{n}, \gamma\right\rangle\right) g_{2}\left(\left\langle\chi \Lambda \backslash\left(O_{1} \cup \ldots \cup O_{n}\right), \gamma\right\rangle\right)=\chi_{\left\{\left|\gamma_{O_{1}}\right|=1, \ldots,\left|\gamma_{O_{n}}\right|=1,\left|\gamma_{\Lambda \backslash\left(O_{1} \cup \cdots \cup O_{n}\right)}\right|=0\right\}}(\gamma) .
$$

Hence, $F(\gamma)$ may be identified with the indicator function $\chi_{S_{n}\left(O_{1} \times \cdots \times O_{n}\right)}\left(x_{1}, \ldots, x_{n}\right)$ on $\tilde{\Lambda}^{n} / S_{n}$. Using a monotone class argument, we get the statement for any indicator function, and then for any measurable bounded function on $\Gamma_{\Lambda}^{(n)}$. 
We denote by $\mathcal{P}$ the set of all continuous polynomials on $\Gamma$, i.e., the set of all finite sums of functions of the form $F(\gamma)=\prod_{i=1}^{n}\left\langle\gamma, \varphi_{i}\right\rangle, \varphi_{i} \in \mathcal{D}, i=1, \ldots, n, n \in \mathbb{N}$, and constants. We preserve the notation $\mathcal{P}$ for the set of all $\mu$-classes of functions from $\mathcal{P}$. Using (2.3) and e.g. [5], we see that $\mathcal{P}$ is a dense subset in $L^{2}(\Gamma, \mu)$. Furthermore, any function from $\mathcal{P}$ is cylinder, and we can easily conclude from Lemma 4.1 that its statement remains true for any function $F \in \mathcal{P}$.

We will now show that $\mathcal{P} \subset \bigcap_{n=1}^{\infty} D\left(\tilde{H}^{n}\right)$. We first make some informal calculations. So, we define:

$$
\begin{aligned}
& H_{1} F(\gamma):=\int z d x \exp [-E(x, \gamma)] D_{x}^{+} F(\gamma), \\
& H_{2} F(\gamma):=\int \gamma(d x) D_{x}^{-} F(\gamma),
\end{aligned}
$$

so that $H=-H_{1}-H_{2}$. Then,

$$
H^{n}=\left(-H_{1}-H_{2}\right)^{n}=(-1)^{n} \sum_{I \subset\{1, \ldots, n\}} \mathcal{H}_{I}^{(n)},
$$

where

$$
\begin{aligned}
\mathcal{H}_{I}^{(n)} & :=\mathcal{H}_{I, 1} \mathcal{H}_{I, 2} \ldots \mathcal{H}_{I, n}, \\
\mathcal{H}_{I, i} & :=\left\{\begin{array}{ll}
H_{1}, & i \in I, \\
H_{2}, & i \notin I,
\end{array} \quad i=1, \ldots, n .\right.
\end{aligned}
$$

Furthermore, by induction, we conclude:

$$
\mathcal{H}_{I}^{(n)}=\sum_{J \subset\{1, \ldots, \max \{i: i \in I\}-1\}} \mathcal{H}_{I, J}^{(n)},
$$

where

$$
\begin{aligned}
& \mathcal{H}_{I, J}^{(n)} F(\gamma)=(-1)^{|I \cap J|}\left(\int m_{I, n}\left(d x_{n}\right) U_{I, J, n, x_{n}} \int m_{I, n-1}\left(d x_{n-1}\right) U_{I, J, n-1, x_{n-1}} \ldots \int m_{I, 1}\left(d x_{1}\right) U_{I, J, 1, x_{1}}\right. \\
& \left.\quad \times \prod_{i \in I} \exp \left[-\sum_{u \in \eta \backslash\left\{x_{s}: s \in I^{c}, s>i\right\}} \phi\left(x_{i}-u\right)\right] \prod_{j=1}^{n} G_{I, J, j}\left(x_{1}, \ldots, x_{n}\right) F(\gamma)\right)\left.\right|_{\eta=\gamma}, \\
& I^{c}:=\{1, \ldots, n\} \backslash I, \\
& m_{I, i}\left(d x_{i}\right):= \begin{cases}z d x_{i}, & i \in I, \\
\gamma\left(d x_{i}\right), & i \in I^{c},\end{cases} \\
& U_{I, J, i, x_{i}}:= \begin{cases}D_{x_{i}}^{+}, & i \in I, i \in J^{c}, \\
D_{x_{i}}^{-}, & i \in I^{c}, i \in J^{c}, \\
\mathrm{id}, & i \in J,\end{cases} \\
& G_{I, J, j}^{(n)}\left(x_{1}, \ldots, x_{n}\right):= \begin{cases}\exp \left[-\sum_{r \in I, r<j} \phi\left(x_{j}-x_{r}\right)\right], & j \in I, j \in J^{c}, \\
1-\exp \left[-\sum_{r \in I, r<j} \phi\left(x_{j}-x_{r}\right)\right], & j \in J, \\
1, & j \in I^{c}, j \in J^{c},\end{cases}
\end{aligned}
$$

For example, let $n=9, I=\{3,4,5,8\}, J=\{6,7\}$. Then,

$$
\begin{aligned}
\mathcal{H}_{I, J}^{(n)} F(\gamma)= & \left(\int \gamma\left(d x_{9}\right) D_{x_{9}}^{-} \int z d x_{8} D_{x_{8}}^{+} \int \gamma\left(d x_{7}\right) \int \gamma\left(d x_{6}\right) \int z d x_{5} D_{x_{5}}^{+}\right. \\
& \times \int z d x_{4} D_{x_{4}}^{+} \int z d x_{3} D_{x_{3}}^{+} \int \gamma\left(d x_{2}\right) D_{x_{2}}^{-} \int \gamma\left(d x_{1}\right) D_{x_{1}}^{-} \exp \left[-\sum_{u \in \eta \backslash\left\{x_{9}\right\}} \phi\left(x_{8}-u\right)\right]
\end{aligned}
$$




$$
\begin{aligned}
& \times \exp \left[-\sum_{u \in \eta \backslash\left\{x_{6}, x_{7}, x_{9}\right\}}\left(\phi\left(x_{5}-u\right)+\phi\left(x_{4}-u\right)+\phi\left(x_{3}-u\right)\right)\right] \\
& \times \exp \left[-\phi\left(x_{8}-x_{5}\right)-\phi\left(x_{8}-x_{4}\right)-\phi\left(x_{8}-x_{3}\right)\right] \\
& \times\left(1-\exp \left[-\phi\left(x_{7}-x_{5}\right)-\phi\left(x_{7}-x_{4}\right)-\phi\left(x_{7}-x_{3}\right)\right]\right) \\
& \times\left(1-\exp \left[-\phi\left(x_{6}-x_{5}\right)-\phi\left(x_{6}-x_{4}\right)-\phi\left(x_{6}-x_{3}\right)\right]\right) \\
& \left.\times \exp \left[-\phi\left(x_{5}-x_{4}\right)-\phi\left(x_{5}-x_{3}\right)-\phi\left(x_{4}-x_{3}\right)\right] F(\gamma)\right)\left.\right|_{\eta=\gamma} .
\end{aligned}
$$

Lemma 4.2. Suppose that the conditions of Theorem 4.1 are satisfied. We then have $\mathcal{P} \subset \bigcap_{n=1}^{\infty} D\left(\tilde{H}^{n}\right)$, and for any $F \in \mathcal{P}, \tilde{H}^{n} F$ is given by formulas (4.1)-(4.4) (in which $H$ is replaced by $\tilde{H}$ ).

Proof. This statement follows from (2.3), Lemma 4.1 and formulas (4.1)-(4.4). Indeed, replace in formulas (4.1)(4.4) $\phi(x)$ by the function $\phi_{n}(x):=\phi(x) \chi_{B(n)}(x), n \in \mathbb{N}$, and take $F \in \mathcal{P}$. Then, the obtained functions become cylindrical. Approximate these by functions as in Lemma 4.1 and let $n \rightarrow \infty$. The rest then easily follows.

Lemma 4.3. Suppose that the conditions of Theorem 4.1 are satisfied. Then, for any $F(\gamma)=\prod_{i=1}^{l}\left\langle\gamma, \varphi_{i}\right\rangle, \varphi_{i} \in \mathcal{D}$, $i=1, \ldots, l, l \in \mathbb{N}$, there exists $t>0$ such that $\sum_{n=0}^{\infty}\left\|\tilde{H}^{n} F\right\|_{L^{2}(\mu)} t^{n} /(2 n) !<\infty$.

Proof. We first derive some estimates. that

Let $f_{1}, \ldots, f_{k}$ be bounded integrable functions on $\mathbb{R}^{d}$. Consider $G(\gamma):=\prod_{i=1}^{k}\left\langle\gamma, f_{i}\right\rangle$. From (2.2), we conclude

$$
\begin{aligned}
\int_{\Gamma} G(\gamma) \mu(d \gamma)= & \sum_{i=1}^{k} \sum_{\substack{\left.A_{1}, \ldots, A_{i}\right): \emptyset \neq A_{j} \subset\{1, \ldots, k\}, j=1, \ldots, i \\
A_{j} \text { 's disjoint, } A_{1} \cup \ldots \cup A_{i}=\{1, \ldots, k\}}} \\
& \times \int_{\left(\mathbb{R}^{d}\right)^{i}} g^{(k)}(\underbrace{x_{1}, \ldots, x_{1}}_{\left|A_{1}\right| \text { times }}, \underbrace{x_{2}, \ldots, x_{2}}_{\left|A_{2}\right| \text { times }}, \ldots, \underbrace{x_{i}, \ldots, x_{i}}_{\left|A_{i}\right| \text { times }}) k_{\mu}^{(i)}\left(x_{1}, \ldots, x_{i}\right) d x_{1} \ldots d x_{i},
\end{aligned}
$$

where $g^{(k)}\left(x_{1}, \ldots, x_{k}\right)=(1 / k !) \sum_{\sigma \in S_{k}} f_{1}\left(x_{\sigma(1)}\right) \ldots f_{k}\left(x_{\sigma(k)}\right)$. By induction, we prove

$$
\sum_{i=1}^{k} \sum_{\substack{\left.A_{1}, \ldots, A_{i}\right): \emptyset \neq A_{j} \subset\{1, \ldots, k\}, j=1, \ldots, i \\ A_{j} \text { 's disjoint, } A_{1} \cup \ldots \cup A_{i}=\{1, \ldots, k\}}} 1 \leqslant 2^{k-1} k !, \quad k \in \mathbb{N} .
$$

Therefore, by (2.3),

$$
\int_{\Gamma}|G(\gamma)| \mu(d \gamma) \leqslant 2^{k-1} \max \{1, \xi\}^{k} k ! \prod_{i=1}^{k} \max \left\{\left\|f_{i}\right\|_{L^{1}},\left\|f_{i}\right\|_{L^{\infty}}\right\} .
$$

Note that, for $\mu$-a.e. $\gamma \in \Gamma$,

$$
\begin{aligned}
& D_{x}^{+} G(\gamma)=\sum_{i=0}^{k-1}\left(\begin{array}{l}
i \\
k
\end{array}\right) \int \gamma\left(d y_{1}\right) \ldots \gamma\left(d y_{i}\right) g^{(k)}(y_{1}, \ldots, y_{i}, \underbrace{x, \ldots, x}_{(k-i) \text { times }}), \\
& D_{x}^{-} G(\gamma)=\sum_{i=0}^{k-1}\left(\begin{array}{l}
i \\
k
\end{array}\right)(-1)^{k-i} \int \gamma\left(d y_{1}\right) \ldots \gamma\left(d y_{i}\right) g^{(k)}(y_{1}, \ldots, y_{i}, \underbrace{x, \ldots, x}_{(k-i) \text { times }}) .
\end{aligned}
$$


We next easily get the following identity:

$$
\sum_{k_{1}=0}^{n}\left(\begin{array}{l}
k_{1} \\
n
\end{array}\right) \sum_{k_{2}=0}^{k_{1}}\left(\begin{array}{l}
k_{2} \\
k_{1}
\end{array}\right) \sum_{k_{3}=0}^{k_{2}}\left(\begin{array}{l}
k_{3} \\
k_{2}
\end{array}\right) \ldots \sum_{k_{m}=0}^{k_{m-1}}\left(\begin{array}{c}
k_{m} \\
k_{m-1}
\end{array}\right)=m^{n}, \quad m, n \in \mathbb{N} .
$$

Recall also the standard estimate

$$
\left(a_{1}+\cdots+a_{n}\right)^{2} \leqslant n\left(a_{1}^{2}+\cdots+a_{n}^{2}\right), \quad a_{1}, \ldots, a_{n} \in \mathbb{R}, n \in \mathbb{N} .
$$

Finally, using condition (P), we get, for any $x, y_{1}, \ldots, y_{k} \in \mathbb{R}^{d}$ and $k \in \mathbb{N}$,

$$
1-\exp \left[-\sum_{i=1}^{k} \phi\left(x-y_{i}\right)\right] \leqslant \sum_{i=1}^{k}\left(1-\exp \left[-\phi\left(x-y_{i}\right)\right]\right) .
$$

Let now $F(\gamma)$ be as in the formulation of the lemma. Fix $\Lambda \in \mathcal{O}_{\mathrm{c}}\left(\mathbb{R}^{d}\right)$ and $C_{5}>0$ such that

$$
\left|f_{1}\left(x_{1}\right) \ldots f_{l}\left(x_{l}\right)\right| \leqslant C_{5} \chi_{\Lambda^{l}}\left(x_{1}, \ldots, x_{l}\right), \quad x_{1}, \ldots, x_{l} \in \mathbb{R}^{d} .
$$

For any sets $I, J \subset\{1, \ldots, n\}$ as in (4.3), we define

$$
n_{1}:=|I \cap J|, \quad n_{2}:=\left|I^{c} \cap J\right|, \quad n_{3}:=\left|I \cap J^{c}\right|, \quad n_{4}:=\left|I^{c} \cap J^{c}\right| .
$$

Notice that $n_{1}+n_{2}+n_{3}+n_{4}=n$. Estimating all the multipliers of the form $\mathrm{e}^{-\phi(\cdot)}$ by 1 , and using (4.5)-(4.10), we get from (4.4)

$$
\begin{aligned}
\left\|\mathcal{H}_{I, J}^{(n)} F\right\|_{L^{2}(\mu)}^{2} \leqslant & \max \{1, z\}^{2 n} C_{5}^{2} \max \left\{1, \int_{\Lambda} d x\right\}^{2(n+l)} \max \{1, \xi\}^{2(n+l)} \max \left\{1, \int_{\mathbb{R}^{d}}(1-\exp [-\phi(x)])\right\}^{2 n} \\
& \times 2^{2\left(l+n_{2}\right)-1}\left(2\left(l+n_{2}\right)\right) !\left(n_{1}+n_{3}\right) !\left(n_{1}+n_{3}\right)^{n_{2}}\left(n_{3}+n_{4}\right)^{l+n_{2}}
\end{aligned}
$$

where the factor $2^{2\left(l+n_{2}\right)-1}\left(2\left(l+n_{2}\right)\right)$ ! is connected with estimate (4.5) and the fact that we get monomials of order $\leqslant l+n_{2}$, the factor $\left(n_{1}+n_{3}\right)$ ! is connected with the application of (4.9) to the terms connected with the set $I$, the factor $\left(n_{1}+n_{3}\right)^{n_{2}}$ is connected with the application of (4.9) to the terms connected with $I^{c} \cap J$, and the factor $\left(n_{3}+n_{4}\right)^{l+n_{2}}$ is connected with the application of (4.6), (4.7) to a monomial of order $\leqslant\left(l+n_{2}\right)$. Using the estimate $(2 k) ! \leqslant 4^{k}(k !)^{2}, k \in \mathbb{N}$, we conclude from (4.11) that there exists a constant $C_{6}>0$, independent of $n, I, J$ and thus depending only on $F$, such that

$$
\left\|\mathcal{H}_{I, J}^{(n)} F\right\|_{L^{2}(\mu)}^{2} \leqslant C_{6}^{n}(n !)^{2} n^{2 n} .
$$

Hence, by (4.1)

$$
\left\|\tilde{H}^{n} f\right\|_{L^{2}(\mu)} \leqslant\left(2 C_{6}\right)^{n / 2} n ! n^{n} .
$$

Estimate (4.12), together with Stirling's formula, easily implies the statement of the lemma, and hence the statement of the theorem.

\section{Spectral gap of the generator}

We first prove a coercivity identity for the gradient $D^{-}$. We note that, for any $\gamma \in \Gamma$ and $F \in \mathcal{F} C_{\mathrm{b}}(\mathcal{D}, \Gamma)$, $\left(D^{-}\right)^{2} F(\gamma)$ is the element of the Hilbert space $T_{\gamma}^{\otimes 2}=L^{2}\left(\left(\mathbb{R}^{d}\right)^{2}, \gamma^{\otimes 2}\right)$ given by $\left(D^{-}\right)^{2} F(\gamma, x, y)=D_{x}^{-} D_{y}^{-} F(\gamma)$, $x, y \in \mathbb{R}^{d}$. Furthermore, for any $x, y \in \gamma$ :

$$
D_{x}^{-} D_{y}^{-} F(\gamma)= \begin{cases}F(\gamma \backslash\{x, y\})-F(\gamma \backslash x)-F(\gamma \backslash y)+F(\gamma), & x \neq y \\ F(\gamma)-F(\gamma \backslash x)=-D_{x}^{-} F(\gamma), & x=y .\end{cases}
$$


Through the natural identification of the elements of $T_{\gamma}^{\otimes 2}$ with linear continuous operators in $T_{\gamma}$, we get:

$$
\operatorname{Tr}\left(D^{-}\right)^{2} F(\gamma)\left(\left(D^{-}\right)^{2} F(\gamma)\right)^{*}=\sum_{x, y \in \gamma}\left(D_{x}^{-} D_{y}^{-} F(\gamma)\right)^{2} .
$$

Here, $\operatorname{Tr}$ denotes the trace of an operator and $\left(\left(D^{-}\right)^{2} F(\gamma)\right)^{*}$ is the adjoint operator of $\left(D^{-}\right)^{2} F(\gamma)$.

Lemma 5.1 (Coercivity identity). Suppose that the conditions of Theorem 4.1 are satisfied. Then, for any $F \in$ $\mathcal{F} C_{\mathrm{b}}(\mathcal{D}, \Gamma)$, we have

$$
\begin{aligned}
\int_{\Gamma}(H F(\gamma))^{2} \mu(d \gamma)= & \int_{\Gamma}\left[\operatorname{Tr}\left(D^{-}\right)^{2} F(\gamma)\left(\left(D^{-}\right)^{2} F(\gamma)\right)^{*}+\sum_{x, y \in \gamma, x \neq y}(\exp [\phi(x-y)]-1)\right. \\
& \times(F(\gamma \backslash\{x, y\})-F(\gamma \backslash x))(F(\gamma \backslash\{x, y\})-F(\gamma \backslash y))] \mu(d \gamma) .
\end{aligned}
$$

Proof. Analogously to (3.6), we get from (2.1):

$$
\begin{aligned}
& \int_{\Gamma} \mu(d \gamma)\left(\int_{\mathbb{R}^{d}} z d x \exp [-E(x, \gamma)] D_{x}^{+} F(\gamma)\right)^{2} \\
& \quad=\int_{\Gamma} \mu(d \gamma) \sum_{x, y \in \gamma, x \neq y} \exp [\phi(x-y)](F(\gamma \backslash\{x, y\})-F(\gamma \backslash x))(F(\gamma \backslash\{x, y\})-F(\gamma \backslash y)) .
\end{aligned}
$$

Next,

$$
\begin{aligned}
& 2 \int_{\Gamma} \mu(d \gamma) \int_{\mathbb{R}^{d}} z d x \exp [-E(x, \gamma)] D_{x}^{+} F(\gamma) \int_{\mathbb{R}^{d}} \gamma(d y) D_{y}^{-} F(\gamma) \\
& =2 \int_{\Gamma} \mu(d \gamma) \int_{\mathbb{R}^{d}} \gamma(d x)(F(\gamma)-F(\gamma \backslash x)) \int_{\mathbb{R}^{d}}(\gamma \backslash x)(d y)(F(\gamma \backslash\{x, y\})-F(\gamma \backslash x)) \\
& =\int_{\Gamma} \mu(d \gamma) \sum_{x, y \in \gamma, x \neq y}[(F(\gamma)-F(\gamma \backslash x))(F(\gamma \backslash\{x, y\})-F(\gamma \backslash x)) \\
& \quad+(F(\gamma)-F(\gamma \backslash y))(F(\gamma \backslash\{x, y\})-F(\gamma \backslash y))] .
\end{aligned}
$$

Finally,

$$
\begin{aligned}
\int_{\Gamma} \mu(d \gamma)\left(\int_{\mathbb{R}^{d}} \gamma(d x) D_{x}^{-} F(\gamma)\right)^{2}= & \int_{\Gamma} \mu(d \gamma) \sum_{x \in \gamma}(F(\gamma \backslash x)-F(\gamma))^{2} \\
& +\int_{\Gamma} \mu(d \gamma) \sum_{x, y \in \gamma, x \neq y}(F(\gamma \backslash x)-F(\gamma))(F(\gamma \backslash y)-F(\gamma)) .
\end{aligned}
$$

By (3.5) and (5.1)-(5.5), the lemma follows.

Theorem 5.1. Suppose that $(\mathrm{P})$ holds, $z>0$, and

$$
\delta:=\int_{\mathbb{R}^{d}}(1-\exp [-\phi(x)]) z d x<1 .
$$

Let $\mu \in \mathcal{G}(z, \phi)$. Then, the set $(0,1-\delta)$ does not belong to the spectrum of $H$. 
Proof. We fix any $F \in \mathcal{F} C_{\mathrm{b}}(\mathcal{D}, \Gamma)$. By (5.1) and (5.2), we have:

$$
\operatorname{Tr}\left(D^{-}\right)^{2} F(\gamma)\left(\left(D^{-}\right)^{2} F(\gamma)\right)^{*} \geqslant \sum_{x \in \gamma}\left(D_{x}^{-} D_{x}^{-} F(\gamma)\right)^{2}=\sum_{x \in \gamma}\left(D_{x}^{-} F(\gamma)\right)^{2}, \quad \gamma \in \Gamma .
$$

Using (P), (2.1), (5.6), and the Cauchy-Schwarz inequality, we next have

$$
\begin{aligned}
& \left|\int_{\Gamma} \sum_{x, y \in \gamma, x \neq y}(\exp [\phi(x-y)]-1)(F(\gamma \backslash\{x, y\})-F(\gamma \backslash x))(F(\gamma \backslash\{x, y\})-F(\gamma \backslash y)) \mu(d \gamma)\right| \\
& \quad \leqslant \int_{\Gamma} \sum_{x, y \in \gamma, x \neq y}(\exp [\phi(x-y)]-1)(F(\gamma \backslash\{x, y\})-F(\gamma \backslash y))^{2} \mu(d \gamma) \\
& \quad=\int_{\Gamma} \mu(d \gamma) \int_{\mathbb{R}^{d}} \gamma(d y) \int_{\mathbb{R}^{d}}(\gamma \backslash y)(d x)(\exp [\phi(x-y)]-1)(F(\gamma \backslash\{x, y\})-F(\gamma \backslash y))^{2} \\
& \quad=\int_{\Gamma} \mu(d \gamma) \int_{\mathbb{R}^{d}} z d y \exp [-E(y, \gamma)] \int_{\mathbb{R}^{d}} \gamma(d x)(\exp [\phi(x-y)]-1)(F(\gamma \backslash x)-F(\gamma))^{2} \\
& \quad=\int_{\Gamma} \mu(d \gamma) \int_{\mathbb{R}^{d}} z d y \int_{\mathbb{R}^{d}} \gamma(d x) \exp [-E(y, \gamma \backslash x)](1-\exp [-\phi(x-y)])(F(\gamma \backslash x)-F(\gamma))^{2} \\
& \leqslant \int_{\Gamma} \mu(d \gamma) \int_{\mathbb{R}^{d}} z d y \int_{\mathbb{R}^{d}} \gamma(d x)(1-\exp [-\phi(x-y)])(F(\gamma \backslash x)-F(\gamma))^{2} \\
& =\int_{\mathbb{R}^{d}}(1-\exp [-\phi(y)]) z d y \times \int_{\Gamma} \mu(d \gamma) \int_{\mathbb{R}^{d}} \gamma(d x)\left(D_{x}^{-} F(\gamma)\right)^{2}=\delta(H F, F)_{L^{2}(\mu)} .
\end{aligned}
$$

Using Lemma 5.1, (5.7), and (5.8), we get, for each $F \in \mathcal{F} C_{\mathrm{b}}(\mathcal{D}, \Gamma)$ :

$$
(H F, H F)_{L^{2}(\mu)} \geqslant(1-\delta)(H F, F)_{L^{2}(\mu)} .
$$

From Theorem 4.1, we then conclude that (5.9) holds true for each $F \in D(H)$. Therefore, denoting by $\left(E_{\lambda}\right)_{\lambda \geqslant 0}$ the resolution of the identity of the operator $H$, we have:

$$
\int_{[0, \infty)} \lambda(\lambda-(1-\delta)) d\left(E_{\lambda} F, F\right)_{L^{2}(\mu)} \geqslant 0, \quad F \in D(H) .
$$

From here the statement of the theorem trivially follows.

Corollary 5.1 (Poincaré inequality). Suppose (P) and (5.6) hold and suppose $\mu$ is an extreme point of the convex set $\mathcal{G}(z, \phi)$. Then,

$$
\mathcal{E}(F, F) \geqslant(1-\delta) \int_{\Gamma}\left(F(\gamma)-\langle F\rangle_{\mu}\right)^{2} \mu(d \gamma), \quad F \in D(\mathcal{E}),
$$

where $\langle F\rangle_{\mu}:=\int_{\Gamma} F(\gamma) \mu(d \gamma)$.

Remark 5.1. The Poincaré inequality (5.10) means that, in addition to the fact that the set $(0,1-\delta)$ does not belong to the spectrum of $H$, we also have that the kernel of $H$ consists only of the constants. 
Proof of Corollary 5.1. Since $\mu$ is extreme in $\mathcal{G}(z, \phi)$, analogously to proof of the part (i) $\Rightarrow$ (ii) of [2, Theorem 6.2], we conclude:

$$
\left\{v \in \mathcal{G}(z, \phi) \mid v=\rho \cdot \mu \text { for some bounded, } \mathcal{B}(\Gamma) \text {-measurable function } \rho: \Gamma \rightarrow \mathbb{R}_{+}\right\}=\{\mu\} .
$$

Let $G \in D(\mathcal{E})$ be such that $\mathcal{E}(G)=0$. It suffices to prove that $G=$ const. By the proof of [2, Lemma 6.1], without loss of generality, we can suppose that the function $G$ is bounded.

Now, we modify the proof of the part (ii) $\Rightarrow$ (iii) of [2, Theorem 6.2]. Since $1 \in \mathcal{F} C_{\mathrm{b}}(\mathcal{D}, \Gamma)$ and $D^{-} 1=0$, replacing $G$ by $G-\operatorname{ess} \inf G$, we may suppose that $G \geqslant 0$, and, in addition, that $\int G d \mu=1$. Define $v:=G \cdot \mu$. Since $\mathcal{E}(G)=0$, by (3.9), we have that $G(\gamma \backslash x)-G(\gamma)=0 \tilde{\mu}$-a.e. Since $\mu \in \mathcal{G}(z, \phi)$, we have, for any measurable function $F: \Gamma \times \mathbb{R}^{d} \rightarrow[0,+\infty]$,

$$
\begin{aligned}
\int_{\Gamma} v(d \gamma) \int_{\mathbb{R}^{d}} \gamma(d x) F(\gamma, x) & =\int_{\Gamma} \mu(d \gamma) \int_{\mathbb{R}^{d}} \gamma(d x) G(\gamma) F(\gamma, x)=\int_{\Gamma} \mu(d \gamma) \int_{\mathbb{R}^{d}} \gamma(d x) G(\gamma \backslash x) F(\gamma, x) \\
& =\int_{\Gamma} \mu(d \gamma) \int_{\mathbb{R}^{d}} z d x \exp [-E(x, \gamma)] G(\gamma) F(\gamma \cup x, x) \\
& =\int_{\Gamma} v(d \gamma) \int_{\mathbb{R}^{d}} z d x \exp [-E(x, \gamma)] F(\gamma \cup x, x) .
\end{aligned}
$$

Hence, $v \in \mathcal{G}(z, \phi)$ and, by (5.11), $G=1 \mu$-a.e.

Let us suppose that the potential $\phi$ satisfies the following condition:

(LAHT) (Low activity-high temperature regime)

$$
\delta=\int_{\mathbb{R}^{d}}(1-\exp [-\phi(x)]) z d x<\exp (-1) .
$$

Under (P) and (LAHT), there exists a unique Gibbs measure $\mu \in \mathcal{G}(z, \phi)$, see [20] and [13, Theorem 6.2] (notice that (2.1) and (P) imply that the correlation functions of any $\mu \in \mathcal{G}(z, \phi)$ satisfy the Ruelle bound (2.3) with $\xi=1$, and hence we can take the constant $C_{R}$ in [13, Theorem 6.2] to be equal to $e$ ). The following statement now immediately follows from Corollary 5.1.

Corollary 5.2 (Poincaré inequality in the LAHT regime). Assume that (P) and (LAHT) are satisfied and consider the unique Gibbs measure $\mu \in \mathcal{G}(z, \phi)$. Then, (5.10) holds.

Remark added during the revision of the paper. Prof. L. Wu has informed us that, independently of our result, he has proved an estimate for the spectral gap of the GD in a finite volume for positive potentials and in the hard core case [24]. In particular, his lower bound for the spectral gap for positive potentials coincide with our estimate of the spectral gap of the GD in infinite volume.

\section{Acknowledgements}

We are grateful to S. Albeverio, T. Pasurek, and M. Röckner for useful discussions. We would also like to thank the referee for a careful reading of the manuscript and making very useful comments and suggestions. Yu.K. gratefully acknowledges the financial support of the DFG through Projects 436 UKR 113/61 and 436 UKR 113/67. E.L. gratefully acknowledges the financial support of SFB 611, Bonn University. 


\section{References}

[1] S. Alberverio, Yu.G. Kondratiev, M. Röckner, Analysis and geometry on configuration spaces, J. Funct. Anal. 154 (1998) $444-500$.

[2] S. Alberverio, Yu.G. Kondratiev, M. Röckner, Analysis and geometry on configuration spaces. The Gibbsian case, J. Funct. Anal. 157 (1998) 242-291.

[3] S. Albeverio, M. Röckner, Dirichlet form methods for uniqueness of martigale problems and applications, in: M.C. Cranston, M.A. Pinsky (Eds.), Stochastic Analysis, in: Proceedings of Symposia in Pure Mathematics, vol. 57, Amer. Math. Soc., 1995, pp. 513-528.

[4] Yu.M. Berezansky, Yu.G. Kondratiev, Spectral Methods in Infinite Dimensional Analysis, Kluwer Academic, Dordrecht, 1994.

[5] Yu.M. Berezansky, Yu.G. Kondratiev, T. Kuna, E. Lytvynov, On a spectral representation for correlation measures in configuration space analysis, Methods Funct. Anal. Topol. 5 (4) (1999) 87-100.

[6] L. Bertini, N. Cancrini, F. Cesi, The spectral gap for a Glauber-type dynamics in a continuous gas, Ann. Inst. H. Poincaré Probab. Statist. 38 (2002) 91-108.

[7] M. Fukushima, Dirichlet Forms and Symmetric Markov Processes, North-Holland, Amsterdam, 1980.

[8] R.A. Holley, D.W. Stroock, Nearest neighbor birth and death processes on the real line, Acta Math. 140 (1987) $103-154$.

[9] O. Kallenberg, Random Measures, Academic Press, 1975.

[10] Yu.G. Kondratiev, Dirichlet operators and the smoothness of solutions of infinite-dimensional elliptic equations, Dokl. Akad. Nauk SSSR 282 (1985) 269-273, (in Russian).

[11] Yu.G. Kondratiev, T. Kuna, Harmonic analysis on configuration spaces I. General theory, Infin. Dimens. Anal. Quantum Probab. Relat. Top. 5 (2002) 201-233.

[12] T. Kuna, Studies in configuration space analysis and applications, PhD Thesis, Bonn University, 1999.

[13] T. Kuna, Properties of marked Gibbs measures in high temperature regime, Methods Funct. Anal. Topol. 7 (3) (2001) 33-53.

[14] Z.-M. Ma, M. Röckner, An Introduction to the Theory of (Non-Symmetric) Dirichlet Forms, Springer-Verlag, 1992.

[15] Z.-M. Ma, M. Röckner, Construction of diffusions on configuration spaces, Osaka J. Math. 37 (2000) 273-314.

[16] X.X. Nguyen, H. Zessin, Integral and differentiable characterizations of the Gibbs process, Math. Nachr. 88 (1979) $105-115$.

[17] C. Preston, Spatial birth-and-death processes, in: Proceedings of the 40th Session of the International Statistical Institute (Warsaw, 1975), vol. 2, in: Bull. Inst. Internat. Statist., vol. 46, 1975, pp. 371-391.

[18] M. Reed, B. Simon, Methods of Modern Mathematical Physics, vol. 2. Fourier Analysis, Self-Adjointness, Academic Press, 1972.

[19] M. Röckner, B. Schmuland, A support property for infinite-dimensional interacting diffusion processes, C. R. Acad. Sci. Paris, Sér. I 326 (1998) 359-364.

[20] D. Ruelle, Statistical Mechanics. Rigorous Results, Benjamins, 1969.

[21] D. Ruelle, Superstable interaction in classical statistical mechanics, Comm. Math. Phys. 18 (1970) 127-159.

[22] D. Surgailis, On multiple Poisson stochastic integrals and associated Markov semigroups, Probab. Math. Statist. 3 (1984) $217-239$.

[23] D. Surgailis, On Poisson multiple stochastic integrals and associated equilibrium Markov processes, in: Theory and Application of Random Fields (Bangalore, 1982), in: Lecture Notes in Control and Inform. Sci., vol. 49, Springer, 1983, pp. 233-248.

[24] L. Wu, Estimate of spectral gap for continuous gas, preprint, 2003. 